\title{
Weiterbildungskurse des Vereins Schweizerischer Geographielehrer im Jahre 1969
}

\author{
Erich Bugmann
}

Die Studienwoche vom 7. bis 11. Juli 1969 wurde bei einer Beteiligung von rund 40 Geographen in Solothurn durchgeführt. Montag und Freitag standen für das Studium der Siedlungsentwicklung in Solothurn und Olten zur Verfügung. Am Dienstag führte eine Exkursion in den Oberaargau und zeigte den Kulturlandschaftswandel im Úbergangsgebiet vom tieferen zum höheren Mittelland. Die Exkursion vom Donnerstag legte einen ausgezeichneten Schnitt vom Fraubrunnenamt bis ins Emmental und in die Gratlagen des Napfgebietes. Damit wurde den Teilnehmern ein reiches Bild des zentralen Mittellandes in seinen Strukturen und Funktionen und in seinem raschen aktuellen Wandel geboten. Am Donnerstag war der Kurs zu Gast bei Professor R. Müller, dem Direktor der Zweiten Juragewässerkorrektion. Nach zwei instruktiven Referaten am Vorabend (Prof. Müller über die technischen Grundlagen und Frl. Dr. Schwab über die archäologischen Forschungsarbeiten) konnte auf einer gut organisierten Besichtigungsfahrt Einblick in die vollendeten und noch laufenden Korrektionsarbeiten genommen werden.

Dank guter Vorbereitung und reicher Dokumentation seitens der Exkursionsleiter profitierten die Teilnehmer sehr für ihren Schulunterricht. Die Kollegen Aerni, Binggeli und Wiesli haben den Stoff des von ihnen betreuten Arbeitstages in den nachstehenden wiedergegebenen Aufsätzen aufgearbeitet. Ein ausgezeichneter Artikel von Prof. Müller über die technischen Grundlagen und die Arbeiten der Zweiten Juragewässerkorrektion ist in "Plan», Schweizerische Zeitschrift für Landes-, Regionalund Ortsplanung Nr. 2, 1963, erschienen. Die von Heinz Rudolf von Rohr dargelegten Aspekte zur Siedlungsentwicklung von Stadt und Region Solothurn sind in erweiterter und vertiefter Form im Band 24 der Mitteilungen der Naturforschenden Gesellschaft des Kantons Solothurn in Druck gegangen.

\section{Gewässer- und Landschaftswandel im Oberaargau}

\author{
Valentin Binggeli
}

Ausgangspunkt des zweiten Exkursionstages war Langenthal, wo in der geographischen Abteilung der neuen Mittelschulen (Seminar, Gymnasium, anhand von Dias und graphischen Darstellungen ein Überblick über Programm und Problemkreis der Veranstaltung gegeben wurde. Der bernische Landesteil Oberaargau ist in einen höhern und tiefern Teil zu gliedern. Den höheren Teil bildet die Vorplatte des Napfgebietes zwischen Huttwil und Herzogenbuchsee/Langenthal, ein Plateau-Hügelland in den marinen Molassesandsteinen, zerschnitten von Kastentälern, die, als fluvioglaziale Rinnen angelegt, heute teils Trockentäler darstellen (z. B. Burgdorf-Wynigen-Langenthal). Im obersten Grenzsaum findet der Übergang zur Fluviallandschaft des Napfs statt.

Der tiefere Oberaargau, beidseits entlang der Aare von der westlichen Kantonsgrenze im Schwemmgebiet der untern Emme zur östlichen Kantonsgrenze an Roth und Murg, ist vorwiegend das Endmoränengebiet (Wangener Stadium) des würmzeitlichen Rhonegletschers mit Moränenhügeln, Seebecken, ausgedehnten Schotterebenen und -terrassen. Vor allem in diesem siedlungs-, verkehrs- und industriereichen Raum häufen sich zwangsläufig die Probleme des Menschen in der Landschaft.

Von der Dachterrasse des Schulgebäudes aus konnten einige aktuelle Fragen anhand von lokalen Beispielen vor Augen geführt werden (Zonenplan, Tangentenstraße, Grundwasserschutzgebiet). Dann warf man einen Blick in die hydrologisch-meteorologische Station der Forschungsstiftung Langenthal; zu einem Teil stammen von ihr die Werte her, die gewässerkundlichen Arbeiten dienen, denen auch die Exkursion Grundlagen verdankte.

Eine kurze Fahrt führte zur Kaltenherberge, von wo aus zu Fuß der Mumenthaler Weiher erreicht wurde. In diesem idyllischen Naturschutzgebiet 

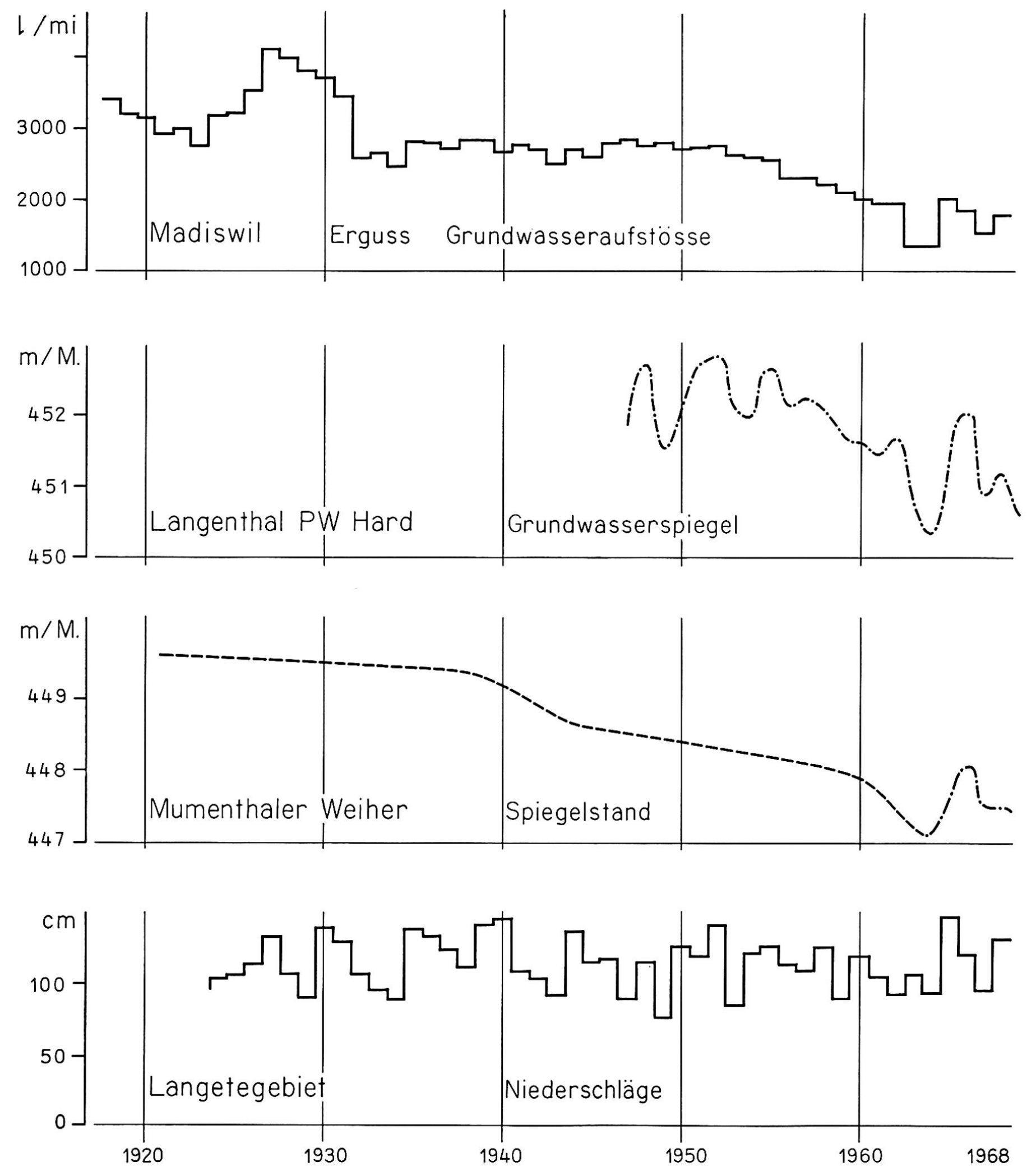

Fig. 1 
zeigt sich am Oberflächengewässer die allgemeine Spiegelsenkung in dem bedeutenden Grundwasserbecken (Fig. 1). Der teils mit dem Grundwasser kommunizierende Weiher leidet an katastrophalem Wassermangel. Dessen Ausmaß ist an der "Strandplatte» zu belegen; der näheren Untersuchung dient ein Pegel des Eidgenössischen Amtes für Wasserwirtschaft.

In der Umgebung des Weihers beobachtet man eine große Reihe von schönen Grundwasseraufstößen (Brunnmatt!), die seit Jahren hydrologisch studiert werden. Die westliche, obere Zone ist fast vollständig versiegt, und Gießenläufe liegen wasserleer. Die Gründe dieses hydrogenen Landschaftswandels sind zahlreich: Wassernutzung in Pumpwerken, Auflassen von Wässermatten, weite Überbauung von Grundwasserarealen (Fig. 2), deren Dach- und Platzwasser in die Kanalisation geführt wird, Waldrodung im Wahlen-Plan des Zweiten Weltkrieges. Die schädigenden Folgen werden einerseits landschaftsschützerisch offenbar, anderseits in den erwähnten Wasserversorgungsanlagen. Eine mögliche Sanierung, beispielsweise durch Erhaltung von Wässermatten (die im Zuge landwirtschaftlichen Strukturwandels weithin aufgegeben und umgebrochen werden zu Ackerland) und durch weitere künstliche Anreicherung des Grundwassers, erheischt großen finanziellen Aufwand.

Die Wanderung nach St. Urban ging unter im unzeitgemäßen "April-Wetter», das den ganzen Tag begleitete. Vom schützenden Auto aus warf man einen Blick in die Wässermatten unterhalb von Langenthal. Diese «ewigen Matten» (Futtergras) des Langentals wurden im Mittelalter durch die Zisterziensermönche von St. Urban angelegt, die im 13. Jahrhundert eine Langeteableitung vornahmen. Das jahrhundertalte Meliorationswerk hat zu einer Bewässerungslandschaft von ganz besonderer Eigenart und Schönheit geführt. Die vom Talflüßchen abzweigenden Wassergräben sind von Bäumen und Buschwerk begleitet, die einer reichen Vogelwelt Unterschlupf gewähren. Als Ganzes haben wir eine harmonische, parkähnliche Kulturlandschaft mit manchen Zügen der ursprünglichen Naturlandschaft vor Augen.

In hervorragender Weise werden von hier aus die Wasserführung der Langete und das Grundwasser reguliert, auch stellen die Matten ein prächtiges Erholungsgebiet der Industriegegend dar. Ihrer starken Gefährdung muß von verschiedenen Blickwinkeln her alle Aufmerksamkeit geschenkt werden.

Als Entgelt für die im Regen untergegangene Wanderung durfte ein Besuch der Klosterkirche St. Urban zählen. Das für die Kulturlandschaftsgeschichte der ganzen Gegend hochbedeutsame Kloster wurde 1194 gegründet. Die heutige Kirche wurde 1711 bis 1730 im Vorarlberger Barock erbaut. Besondere kunsthistorische Bedeutung kommt dem einzigarti-

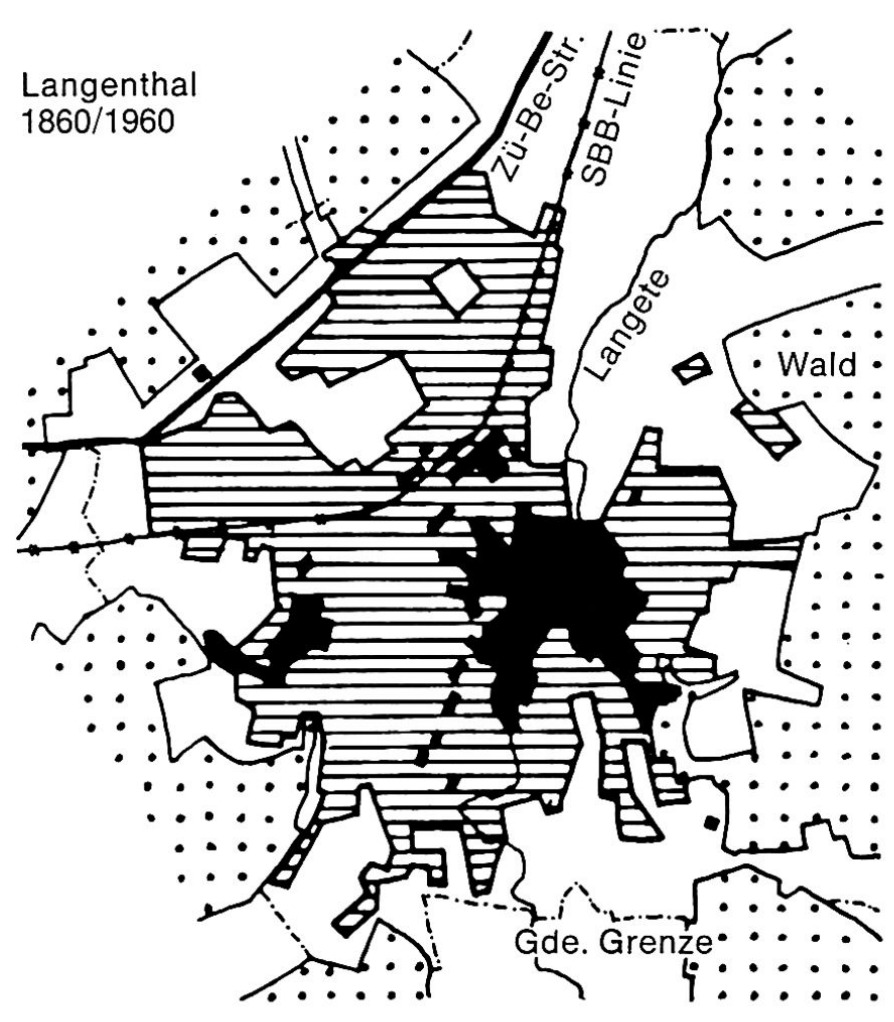

Fig. 2. Räumlicher Ausdehnungsvergleich Langenthals

gen geschnitzten Chorgestühl aus dem Anfang des 18. Jahrhunderts $\mathrm{zu}$, dem "reichsten und künstlerisch vollendetsten Werk dieser Art aus der Zeit des Barocks in der Schweiz» (H. Meyer-Rahn).

Nach dem Mittagessen in Melchnau ergriff Herr Alt-Großrat Johann Flückiger, Landwirt, das Wort. Als Präsident der Flurgenossenschaft war er in den Jahren nach dem Zweiten Weltkrieg die Triebfeder der Melchnauer Güterzusammenlegung. Die Bevölkerung von Melchnau war damals zu 30\% in der Landwirtschaft beschäftigt. Die Zusammenlegung verminderte die Parzellenzahl von 4,7 auf 1,7 je Betrieb, deren es 169 gab (Fig. 3/4). Der erfaßte Perimeter betrug 1200 ha für die Entwässerung und 800 ha für die Zusammenlegung. Die Gesamtkosten beliefen sich auf fast 2 Millionen Franken, woran von Bund und Kanton $75 \%$ geleistet wurden. An die lehrreichen Ausführungen des Praktikers und begeisterten Befürworters schlossen sich Besichtigung von Fluren und eines Hofes an, wo die Theorie in der Ausführung erörtert werden konnte. Auch hier konnten die Bauersleute nur positive Resultate der Melioration anführen. - Die Folgen von Bachkorrektur und Aufhebung der Wässermatten standen hier nicht zur Diskussion.

Von Melchnau führte die Carfahrt durch den höheren Oberaargau nach Madiswil und Leimiswil und in die Buchsiberge. Diese siedlungs- und wirt- 


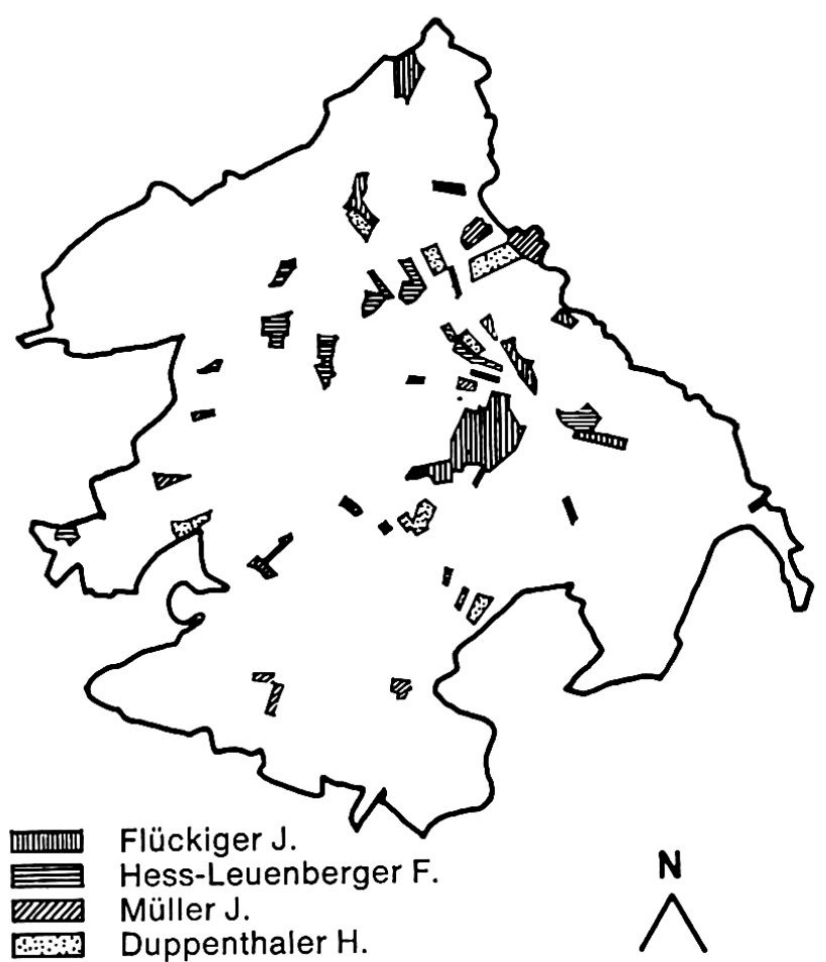

Fig. 3 Alter Zustand

schaftsgeographische Übergangslandschaft zum Emmental ist die Heimat Maria Wasers, die sie im Roman "Land unter Sternen» in meisterhafter dichterischer Schau charakterisiert hat. Sie war auch die Wahlheimat von Cuno Amiet, der sie farbenreich durch sechs Jahrzehnte hindurch festzuhalten verstand. Kulturgeographischen Höhepunkt bildete der Besuch in seinem Atelier, das heute noch - Amiet starb 1961 - wie zu Lebzeiten des Künstlers besteht.

Als weiterer Höhepunkt und gleichsam Abschluß des Tages darf die Fahrt in die solothurnische Enklave Steinhof bezeichnet werden, jene glaziologisch einzigartige Stätte: hier liegen Hunderte von hausgroßen Findlingen aus der südlichen Randmoräne des Würm-Rhone-Gletschers, darunter der größte Erratiker des Mittellandes. Steinhof/Steinenberg findet sich als Objekt Nr. 94 im «Inventar der zu erhaltenden Landschaften und Naturdenkmäler von nationaler Bedeutung».

\section{Literatur}

Aerni, K.: Die Güterzusammenlegung Attiswil. Schulpraxis, Bern 1965.

Bieri W.: Die Wässermatten von Langenthal. Mitt. Natf. Ges. Bern 1949.

Brönnimann, F.: Aus der Urwelt des Oberaargaus. Jahrbuch des Oberaargaus. 1958.

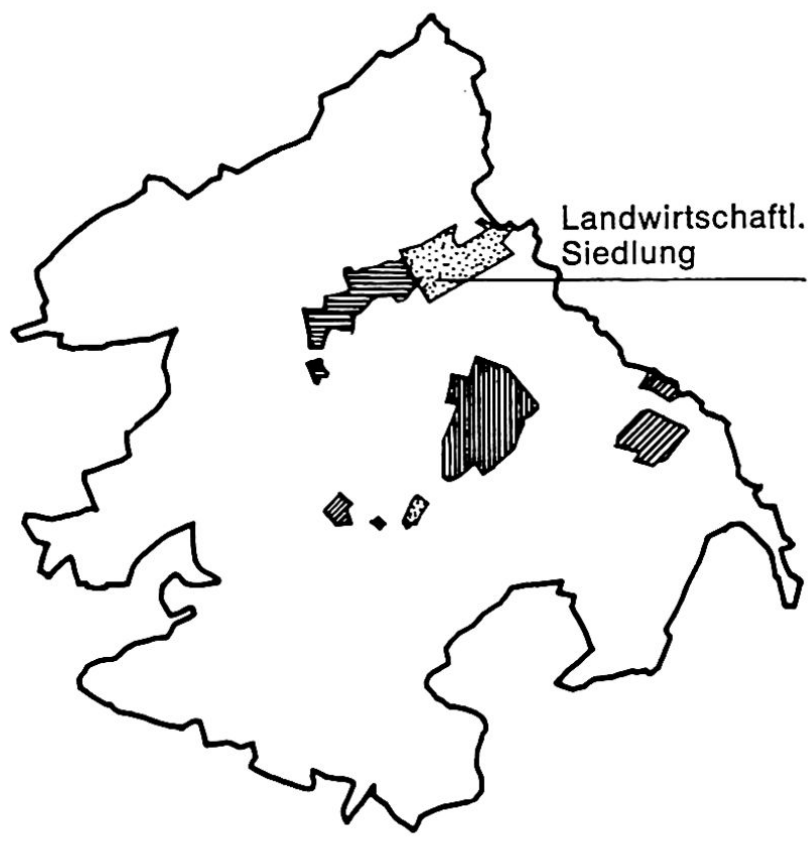

Fig. 4 Neuer Zustand

Flückiger J.: Die Güterzusammenlegung von Melchnau. Jahrbuch des Oberaargaus, Langenthal 1959.

Bericht über die Gesamtmelioration Ersigen-Oesch. Ersigen 1949.

Grosjean, G.: Planungsgrundlagen Region Burgdorf. Stadtverwaltung Burgdorf und Institut für angewandte Geographie der Universität Bern 1968. Meyer, J. R.: Langenthal. Berner Heimatbuch. Bern 1958.

Schmalz, K. L.: Das Findlingsreservat SteinhofSteinenberg in heimatkundlicher Darstellung. Jahrbuch des Oberaargaus. Langenthal 1966.

Zimmermann, H.W.: Die Eiszeit im westlichen zentralen Mittelland. Diss. Zürich 1963.

Zimmermann, H. W.: Zur Landschaftsgeschichte des Oberaargaus. Jahrbuch des Oberaargaus. Langenthal 1969.

\section{Schriften des Berichtverfassers}

Úber Begriff und Begrenzung der Landschaft Oberaargau. Jahrbuch des Oberaargaus. Langenthal 1962.

Die Landschaft im Lichte der Flurnamen. Langenthaler Heimatblätter, Langenthal 1961.

Die geschützten Naturdenkmäler des Oberaargaus. Jahrbuch des Oberaargaus. Langenthal 1965.

Niederschlag und Abfluß im Langetegebiet. Jahrbuch des Oberaargaus. Langenthal 1968. 